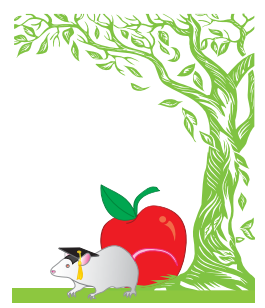

\title{
Lab Animal Management syllabus, part 2
}

In last month's column, I presented the first part of my syllabus from my Laboratory Animal Management and Rules and Regulations course for undergraduate veterinary technician students. Below is the content of the remaining six weeks.

Because my students work with companion animals in veterinary clinics and may not have a proclivity to laboratory animal science (LAS), I look for ways to engage their interest. I invite faculty members and graduate students to talk about their research happening on campus. Visits from the Veterinary Medical Officer or representatives of the Association for Assessment and Accreditation of Laboratory Animal Care International become topics of discussion. During our own semi-annual facility inspections, students may serve as scribes. I play current videos and segments from YouTube, Discovery Channel, National
Public Radio, etc. about LAS to emphasize current events. I bring in newspaper and magazine articles as well as posts from pertinent list-serves (CompMed, Techlink, transgenic, IACUC forums, etc.). To encourage students to make connections between animals and LAS, I ask them to look for stories such as an intriguing case at the vet hospital, a birth at the zoo or a wildlife management practice at a national park. There is an assignment (to earn 'participation points,' as I call them) to submit a summary and personal reflection on ten of these stories. During class breaks, I provide animal-shaped treats like gummy bears and circus cookies.

I incorporate various sources of information about LAS, using Management of Laboratory Animal Care and Use Programs (CRC Press, Boca Raton, FL, 2001) as the primary textbook. Reading assignments in the table below refer to this text. Assessment is done with weekly quizzes and a final exam, in addition to participation points. Because the material is mostly new to students (although some may have worked in the campus lab animal facility (LAF)), I make sure to include time for discussion. Quizzes are graded immediately in class so that we can review the material together.

Each 3-hour class starts with a quiz, presents material in slide format and incorporates demonstrations and other audio-visual teaching modalities. The protocol assignment is the scaffold for the other activities. The concept is to integrate fact and task, relating them to current LAS practices to prepare students for a career in the field, where they will, of course, learn more.

I invite you to take ideas from my experiences teaching lab animal science and to share your own. I may compile them as additional 'fruits of education' in this column.

$\begin{array}{ll}\text { Week } & \text { Presentation topic and material highlights } \\ 5 & \text { Review } \\ & \text { Regulations; purpose of the protocol }\end{array}$

$6 \quad$ Managing quality in the LAF and in research

Quality control and assurance; documentation; sampling; site visits; inspections; staff evaluation; Good Laboratory Practices; disaster planning

$7 \quad$ Managing to keep the LAF clean and safe

Animal Biosafety Levels (ABSL) 1-4; waste management; personal protective equipment; disinfection, sterilization; zoonoses; sentinels; ergonomics; chemical, biological and physical hazards

8 Procurement and fiscal management in the LAF

Budget; cost centers; direct and indirect costs; funding and granting agencies; costs of doing things right and of doing them wrong; costs in time, effort and money to ship animals

$9 \quad$ Managing people

Research team; basic personnel management; researchers as customers; labor laws; planning, organizing, directing, monitoring and staffing the LAF; training, certification and growth; cultural diversity

10 Managing the physical plant

Types of facilities (centralized, barrier, core, etc.); micro- and macroenvironments; caging; feed, bedding, water, enrichment; temperature, humidity, ventilation; noise, lighting, pest control; security; support areas like surgery, receiving, office, cagewash and 'cores' Acronyms, multiple-choice, true/false and short-answer essay, questions; answers must be hand-written

\section{Class activities and assignments}

Finish draft of protocol; conduct a mock IACUC meeting (students exchange their protocols and review them as vet, principal investigator (PI), community member); listen to another PI presentation

Write a standard operating procedure; design a recordkeeping form; skim chs. 7,8 and 14

Submit final protocol; review cagewash cleaning agents; find research products to keep the facility, people and animals on the protocol protected; complete campus occupational health forms; watch Griffin Foundation video on ABSL 1-3 ("Working Safely with Laboratory Animals"); skim ch. 13

Prepare a purchase order of 12-15 animal-related items needed for the protocol; calculate per diem rate for study animal; demonstration of shipping/transporting animals; skim ch. 10

Review real job descriptions and write one for the protocol; write a personal résumé to apply for a vet technician position; discuss Maslow's hierarchy: what is "needed", what motivates; watch American Association for Laboratory Animal Science DVD on careers in lab animal science ("Accept the Challenge to Care"); skim chs. 1, 2 and 3 Watch Uniformed Services University of the Health Sciences video on potential facility problems ("An Exercise in Evaluation of Laboratory Animal Management Practices"); design an appropriate LAF for the animal study; skim chs. 9 and 15

Bring and use Guide for the Care and Use of Laboratory Animals (National Academies Press, Washington, DC, 2011) 